\title{
Modelling of Deuterium Chemistry in Star-Forming Regions
}

\author{
Helen Roberts \\ University of Manchester, School of Physics and Astronomy, \\ Sackville Street, Manchester, M60 1QD, UK \\ email: helen.h.roberts@manchester.ac.uk
}

\begin{abstract}
Several new multiply deuterated species have been detected over the past three years, including $\mathrm{ND}_{3}$ (van der Tak et al. 2002; Lis et al. 2002), $\mathrm{CHD}_{2} \mathrm{OH}, \mathrm{CD}_{3} \mathrm{OH}$ (Parise et al. 2002, 2004), $\mathrm{D}_{2} \mathrm{~S}$ (Vastel et al. 2003), $\mathrm{HD}_{2}^{+}$(Vastel et al. 2004) and $\mathrm{D}_{2} \mathrm{CS}$ (Marcelino et al. 2005). In addition, mono-deuterated species have been observed with abundances $>10 \%$ of their undeuterated analogues (e.g. $\mathrm{CH}_{2} \mathrm{DOH}$ observed by Parise et al. $2002 ; \mathrm{NH}_{2} \mathrm{D}$ observed by Saito et al. 2000 and Hatchell 2003). These are remarkable results, given that the underlying abundance of deuterium in the local interstellar medium (ISM) is $\sim 10^{-5}$ times lower than that of hydrogen (Linsky 1998; Sonneborn et al. 2000).

Such large enhancements in the abundances of deuterium-bearing molecules can either be due to gas-phase or to grain-surface fractionation. Grain-surface reactions are undoubtedly important in producing saturated species such as methanol, water, ammonia, and hydrogen sulphide. Water ice is observed to be abundant and ubiquitous throughout the ISM, and enhanced abundances of gas-phase $\mathrm{NH}_{3}, \mathrm{CH}_{3} \mathrm{OH}, \mathrm{H}_{2} \mathrm{CO}$ and $\mathrm{H}_{2} \mathrm{~S}$ (among others) are observed in warmer regions around protostars where grain mantles have evaporated.

Recent observational and theoretical evidence suggests that the deuterium fractionation in star-forming regions is set by gas-phase and grain-surface reactions during the cold, dense preprotostellar phase. For species which form on grain surfaces via $\mathrm{H}$ atom addition to $\mathrm{CO}, \mathrm{N}, \mathrm{O}$ and $\mathrm{S}$, the deuterium fractionation on grains comes from the relative amounts of atomic $\mathrm{D}$ and $\mathrm{H}$ which are accreting from the gas. The observations of deuterated methanol and $\mathrm{D}_{2} \mathrm{~S}$ require that the gas-phase atomic $\mathrm{D} / \mathrm{H}$ ratio at the time the molecules formed was $\geqslant 0.1$.

This paper presents results from chemical models of the prestellar core phase of star formation, showing how this high atomic $\mathrm{D} / \mathrm{H}$ ratio can be produced, and discusses how models can also be used to look at deuterium fractionation in the protostellar stages of star formation.
\end{abstract}

Keywords. ISM: abundances — ISM: molecules — molecular processes — stars: formation

\section{Introduction}

Observations of deuterated molecules are extremely useful probes of the physical conditions in astronomical objects. Abundances of deuterated molecular ions can be used to determine the ionisation fraction in dark clouds and prestellar cores, a parameter which regulates the collapse of magnetised gas, and, thus, is important in models of star formation. Where ices have evaporated and released the products of grain surface chemistry (around new protostars or in cometary comae), deuterium fractionation can provide valuable information on the temperatures under which the ices formed and how species have been processed within them. The underlying abundance of deuterium is an important cosmological parameter, but it cannot be directly determined outside of the local ISM; observations of deuterated molecules, however, can be made throughout the Galaxy, and beyond, and used to infer the underlying $\mathrm{D} / \mathrm{H}$ ratio. 
Table 1. A sample of the molecular $\mathrm{D} / \mathrm{H}$ ratios in selected sources.

\begin{tabular}{lcccccc}
\hline & TMC-1 CP & L134N & L1544 & Barnard 1 & NGC1333 IRAS4A & IRAS 16293-2422 \\
$\mathrm{DCO}^{+}$ & $0.02^{1}$ & $0.18^{1}$ & $0.04^{12}$ & - & $0.01^{19}$ & $0.01^{22}$ \\
$\mathrm{~N}_{2} \mathrm{D}^{+}$ & $0.08^{1}$ & $0.3^{10}$ & $0.2^{12}$ & $0.15^{10}$ & - & - \\
$\mathrm{NH}_{2} \mathrm{D}$ & $0.01^{1}$ & $0.1^{1}$ & $0.13^{13}$ & $0.07^{16}$ & $0.07^{13}$ & $0.1^{22}$ \\
$\mathrm{NHD}_{2}$ & - & $0.05^{11}$ & - & - & - & - \\
$\mathrm{ND}_{3}$ & - & - & - & $0.001^{17}$ & $0.001^{20}$ & - \\
$\mathrm{DCN}$ & $0.023^{2}$ & $0.05^{5}$ & - & - & - & - \\
$\mathrm{DNC}$ & $0.015^{3}$ & - & $0.03^{14}$ & - & - & - \\
$\mathrm{HDO}$ & - & - & - & - & - & $0.03^{23}$ \\
$\mathrm{HDCO}$ & - & $0.07^{5}$ & - & $0.14^{18}$ & - & $0.15^{22}$ \\
$\mathrm{D}_{2} \mathrm{CO}$ & - & - & $0.04^{15}$ & $0.06^{18}$ & $0.07^{21}$ & $-0.13^{24}$ \\
$\mathrm{HDCS}$ & $0.02^{4}$ & - & - & $0.3^{18}$ & - & - \\
$\mathrm{D}_{2} \mathrm{CS}$ & - & - & - & $0.1^{18}$ & - & $0.015^{25}$ \\
$\mathrm{CH} \mathrm{H}_{3} \mathrm{OD}$ & $0.026^{5}$ & $<0.03^{5}$ & - & - & - & $0.3^{25}$ \\
$\mathrm{CH} \mathrm{H}_{2} \mathrm{DOH}$ & - & - & - & - & - & $0.06^{25}$ \\
$\mathrm{CHD} \mathrm{D}_{2} \mathrm{OH}$ & - & - & - & - & - & - \\
$\mathrm{CD}_{3} \mathrm{OH}$ & - & - & - & - & - & - \\
$\mathrm{C}_{2} \mathrm{D}$ & $0.01^{6}$ & - & - & - & - & - \\
$\mathrm{C}_{4} \mathrm{D}$ & $0.004^{7}$ & - & - & - & - & - \\
$\mathrm{DC}_{3} \mathrm{~N}$ & $0.03-0.1^{8}$ & - & - & - & - & - \\
$\mathrm{DC}_{5} \mathrm{~N}$ & $0.013^{9}$ & - & - & - & - & - \\
\hline
\end{tabular}

References: ${ }^{1}$ Tiné et al. $2000 ;{ }^{2}$ Wootten $1987 ;{ }^{3}$ Guélin et al. $1982 ;{ }^{4}$ Minowa et al. $1997 ;{ }^{5}$ Turner 2001; ${ }^{6}$ Millar et al. $1989 ;{ }^{7}$ Turner $1989 ;{ }^{8}$ Howe et al. $1994 ;{ }^{9}$ Macleod et al. $1981 ;{ }^{10}$ Gerin et al. 2001; ${ }^{11}$ Roueff et al. 2000; ${ }^{12}$ Caselli et al. $2002 ;{ }^{13}$ Shah \& Wootten $2001 ;{ }^{14}$ Hirota et al. 2003; ${ }^{15}$ Bacmann et al. $2002 ;{ }^{16}$ Saito et al. $2000 ;{ }^{17}$ Lis et al. $2002 ;{ }^{18}$ Marcelino et al. $2005 ;{ }^{19}$ Stark et al. 1999; ${ }^{20}$ van der Tak et al. 2002; ${ }^{21}$ Loinard et al. $2002 ;{ }^{22}$ van Dishoeck et al. $1995 ;{ }^{23}$ Parise et al. 2005; ${ }^{24}$ Ceccarelli et al. $2001 ;{ }^{25}$ Parise et al. 2004.

These studies rely on our understanding of the chemical routes leading to molecular deuteration. Over the past few years, the observations of deuterated species with abundances many orders of magnitude higher than expected have led to renewed interest in the subject of deuterium fractionation and a re-examination of the chemical models.

Table 1 shows abundances of deuterated species relative to their completely hydrogenated analogues for a variety of interstellar and protostellar sources. TMC-1 is the archetypal dark cloud; L134N was also thought to be a dark cloud, but recent observations (Pagani et al. 2005) suggest it may have more in common with prestellar cores like L1544 and Barnard 1. NGC1333 IRAS4A and IRAS 16293-2422 are warmer, low-mass protostellar sources.

The paper is arranged as follows: $\S 2$ explains the basic deuterium fractionation processes and shows how molecular $\mathrm{D} / \mathrm{H}$ ratios in cold clouds can be estimated, $\S 3$ presents results from prestellar core models, showing how high deuteration fractionation is achieved, while $\S 4$ discusses the ratios observed in protostellar sources.

Throughout, $[\mathrm{X}]$ denotes the fractional abundance of species $\mathrm{X}$ with respect to $\mathrm{H}_{2}$, $N(\mathrm{X})$ is the column density of $\mathrm{X}$, and 'molecular $\mathrm{D} / \mathrm{H}$ ratio' refers to the ratio of a deuterated species abundance to that of its totally hydrogenated analogue.

\section{Dark Clouds}

The enhanced abundances of deuterated species in interstellar dark clouds such as TMC-1, which have temperatures of $10 \mathrm{~K}$ and $\mathrm{H}_{2}$ densities of $10^{4} \mathrm{~cm}^{-3}$, are typically on the order of a few percent (see Table 1). It has long been understood (see e.g. Millar et al. 1989) that these are due to chemical fractionation. A full list of the fractionation 
reactions used in the models can be found in Roberts \& Millar (2000) and Roberts et al. (2004), but a selection that will be referred to in this paper are listed below.

$$
\begin{aligned}
\mathrm{H}_{3}^{+}+\mathrm{HD} & \rightleftharpoons \mathrm{H}_{2} \mathrm{D}^{+}+\mathrm{H}_{2}+220 \mathrm{~K} \\
\mathrm{H}_{2} \mathrm{D}^{+}+\mathrm{HD} & \rightleftharpoons \mathrm{HD}_{2}^{+}+\mathrm{H}_{2}+187 \mathrm{~K} \\
\mathrm{HD}_{2}^{+}+\mathrm{HD} & \rightleftharpoons \mathrm{D}_{3}^{+}+\mathrm{H}_{2}+234 \mathrm{~K} \\
\mathrm{CH}_{3}^{+}+\mathrm{HD} & \rightleftharpoons \mathrm{CH}_{2} \mathrm{D}^{+}+\mathrm{H}_{2}+370 \mathrm{~K} \\
\mathrm{CH}_{2} \mathrm{D}^{+}+\mathrm{HD} & \rightleftharpoons \mathrm{CHD}_{2}^{+}+\mathrm{H}_{2}+370 \mathrm{~K}
\end{aligned}
$$

Fractionation occurs because of the exothermicity of the forward reactions, so if the rate coefficient for reaction $(x)$ is $k_{(x)}$, the rate for the reverse reaction is $k_{(x)} \mathrm{e}^{-\Delta E / T}$, where $\Delta E$ is the energy barrier, in $\mathrm{K}$, as listed for each reaction.

The molecular ion, $\mathrm{H}_{3}^{+}$, plays a crucial role the ion-molecule chemistry which dominates in dark clouds (see e.g. Herbst 2000), while the bulk of the deuterium in the cloud is assumed to be incorporated into HD. As reaction (2.1) shows, these two species react efficiently to form $\mathrm{H}_{2} \mathrm{D}^{+}$and $\mathrm{H}_{2}$. Due to the energy barrier, however, the reverse reaction is very slow for $T \leqslant 30 \mathrm{~K}$ so $\left[\mathrm{H}_{2} \mathrm{D}^{+}\right]$becomes enhanced relative to $\left[\mathrm{H}_{3}^{+}\right]$. This enhancement can be estimated via the equation:

$$
\frac{\left[\mathrm{H}_{2} \mathrm{D}^{+}\right]}{\left[\mathrm{H}_{3}^{+}\right]}=\frac{k_{(2.1)}}{k_{(2.1)} \exp (-220 / \mathrm{T})+k_{(2.2)}[\mathrm{HD}]+k_{C O}[\mathrm{CO}]+k_{d . r .}\left[\mathrm{e}^{-}\right]} \frac{[\mathrm{HD}]}{\left[\mathrm{H}_{2}\right]}
$$

As well as being destroyed by reactions $(2.1)$ and $(2.2), \mathrm{H}_{2} \mathrm{D}^{+}$can also react via proton transfer to neutral species (represented here by CO, as it is the most abundant of them) and by dissociative recombination (d.r.) with electrons.

In dark clouds, at $10 \mathrm{~K}, k_{(2.1)} \exp (-220 / T) \sim 10^{-18} \mathrm{~cm}^{3} \mathrm{~s}^{-1}$, while the fractional abundance of HD is $3 \times 10^{-5}$ and $k_{(2.2)}=8 \times 10^{-10} \mathrm{~cm}^{3} \mathrm{~s}^{-1}$, so these are minor channels. Destruction is dominated by $\mathrm{CO}\left([\mathrm{CO}] \sim 10^{-4} ; k_{C O} \sim 10^{-9} \mathrm{~cm}^{3} \mathrm{~s}^{-1}\right)$ and electrons $\left(\left[\mathrm{e}^{-}\right] \sim\right.$ $2 \times 10^{-7} ; k_{\text {d.r. }} \sim 3 \times 10^{-7} \mathrm{~cm}^{3} \mathrm{~s}^{-1}$ ), which gives an enhancement factor of $\sim 10^{4}$ and an $\mathrm{H}_{2} \mathrm{D}^{+} / \mathrm{H}_{3}^{+}$ratio of $\sim 0.1$. When $\mathrm{H}_{3}^{+}$and $\mathrm{H}_{2} \mathrm{D}^{+}$then react the assumption is that, if there is more than one place for the $\mathrm{D}$ to end up in the products, the branching between the different channels will be statistical; e.g. for the reaction of $\mathrm{CO}$ with $\mathrm{H}_{2} \mathrm{D}^{+}$there is twice as much chance to transfer a proton and form $\mathrm{HCO}^{+}$, so $\mathrm{DCO}^{+/} \mathrm{HCO}^{+} \sim \frac{1}{3} \mathrm{H}_{2} \mathrm{D}^{+/} \mathrm{H}_{3}^{+}$. Similar approximations can be made for other molecules, explaining the fractionation of a few percent which is typically observed towards TMC-1.

\section{Prestellar Cores}

So how can ratios significantly higher than this be produced? In recent years submm cameras like SCUBA and SHARC have allowed observers to directly map the dust distribution in dense clouds and compare this to molecular abundances. In an increasing number of sources, key tracer molecules such as CO and CS are found to be heavily depleted at the dust peak. The best explanation for this is that the molecules are freezing onto the grain surfaces. Depletion of CO has been observed in L134N (Pagani et al. 2005) and L1544 (Caselli et al. 1999), sources which also have high molecular D/H ratios (see Table 1).

It has long been predicted that freeze-out of neutral species such as CO will enhance molecular D/H ratios (Brown \& Millar 1989). This is clear from eq. (2.6): if [CO] is reduced, the enhancement factor increases. In prestellar cores the ionisation fraction is also expected to be lower than in dark clouds, as the higher densities mean collisions 

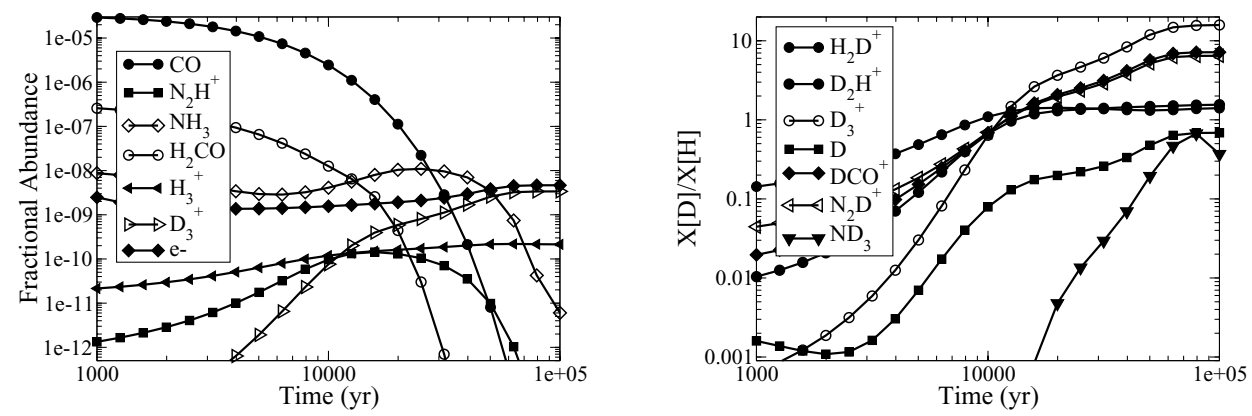

Figure 1. Predicted evolution of fractional abundances (left) and molecular D/H ratios (right) for selected species, predicted by an accretion model at $8 \mathrm{~K}, \mathrm{n}\left(\mathrm{H}_{2}\right)=10^{6} \mathrm{~cm}^{-3}$.

leading to recombination reactions happen more frequently. Observations of molecular ions in L1544 suggest $\left[\mathrm{e}^{-}\right] \sim$ a few times $10^{-9}$ (Caselli et al. 2002). The second term in the denominator in eq. (2.6), reaction (2.2), then becomes the dominant destruction term, and limits the enhancement in $\mathrm{H}_{2} \mathrm{D}^{+} / \mathrm{H}_{3}^{+}$. Reaction (2.2) forms $\mathrm{HD}_{2}^{+}$, however, which can also pass on its fractionation to other neutral species (and more efficiently than $\mathrm{H}_{2} \mathrm{D}^{+}$since, statistically, it will transfer a deuteron $\frac{2}{3}$ of the time), so one could write an equation for enhancement of $\mathrm{HD}_{2}^{+} / \mathrm{H}_{3}^{+}$very similar to eq. (2.6). Once most of the CO has disappeared from the gas and with $\left[\mathrm{e}^{-}\right] \sim 3 \times 10^{-9}$ the enhancement is $\sim 10^{5}$ : $\left[\mathrm{HD}_{2}^{+}\right] \sim\left[\mathrm{H}_{2} \mathrm{D}^{+}\right] \sim\left[\mathrm{H}_{3}^{+}\right]$.

Reaction (2.3) of $\mathrm{HD}_{2}^{+}$with $\mathrm{HD}$ forms $\mathrm{D}_{3}^{+}$. Again, there is a barrier in the reverse direction, and, obviously, no further exchange with $\mathrm{HD}$ can occur, so in the prestellar cores where the $\mathrm{CO}$ and electron abundances are low, the $\mathrm{D}_{3}^{+} / \mathrm{H}_{3}^{+}$ratio can become very large. In the limit where all the CO has frozen out:

$$
\frac{\mathrm{D}_{3}^{+}}{\mathrm{H}_{3}^{+}} \sim \frac{k_{(2.3)}}{k_{d . r .}\left[\mathrm{e}^{-}\right]} \frac{\mathrm{HD}}{\mathrm{H}_{2}} \sim 20,
$$

but even before all the neutral species freeze-out, the $\mathrm{D}_{3}^{+} / \mathrm{H}_{3}^{+}$ratio is many times higher than the $\mathrm{HD} / \mathrm{H}_{2}$ ratio (and when $\mathrm{D}_{3}^{+}$reacts it always produces a deuterated product), leading to high fractionation in other species.

This is illustrated in Figure 1, which shows results from a chemical model which includes multiple deuteration, gas-phase reactions, and freeze-out of species onto grains (see Roberts et al. 2003, 2004 for full details). The temperature is $8 \mathrm{~K}$ and the $\mathrm{H}_{2}$ density is $10^{6} \mathrm{~cm}^{-3}$. The molecular $\mathrm{D} / \mathrm{H}$ ratios increase over time, while the abundances of $\mathrm{CO}$ and other heavy species decrease as they accrete onto the grains. It is assumed that $\mathrm{H}_{2}, \mathrm{HD}$ and $\mathrm{D}_{2}$ are formed on the grains and returned to the gas-phase, so at the end-point of the model only species composed entirely of $\mathrm{H}$ and $\mathrm{D}$ (along with $\mathrm{He}, \mathrm{He}^{+}$ and electrons) remain in the gas-phase. After $10^{5} \mathrm{yr}$ this model predicts that $\mathrm{D}_{3}^{+}$will be the dominant ion: $\left[\mathrm{D}_{3}^{+}\right] \sim\left[\mathrm{e}^{-}\right] \sim$ a few times $10^{-9}$. It also agrees with our simplified estimates from eqs. (2.6) and (3.1) that $\mathrm{H}_{2} \mathrm{D}^{+} / \mathrm{H}_{3}^{+} \sim \mathrm{HD}_{2}^{+} / \mathrm{H}_{3}^{+} \sim 1$ and $\mathrm{D}_{3}^{+} / \mathrm{H}_{3}^{+} \sim 20$.

In L1544, $\mathrm{H}_{2} \mathrm{D}^{+}$and $\mathrm{HD}_{2}^{+}$have been detected (by Caselli et al. 2003 and Vastel et al. 2004, respectively), and their abundance determinations are in agreement with model predictions. The models suggests that $\mathrm{D}_{3}^{+}$is the best ion to trace the ionisation fraction of a prestellar core in the last stages of collapse. $\mathrm{As}_{3}^{+}$is a symmetrical molecule, however, it does not have a sub-mm spectrum and, thus, cannot be observed in these regions. Determining the relative abundances of $\mathrm{H}_{2} \mathrm{D}^{+}$and $\mathrm{HD}_{2}^{+}$, and using a detailed 

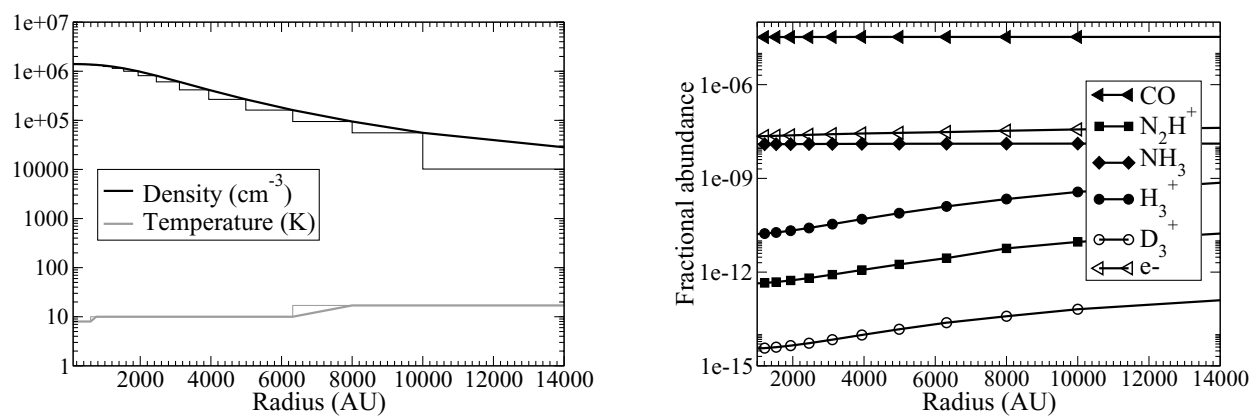

Figure 2. Left: Physical parameters of L1544 as a function of radius (Tafalla et al. 2002), along with the model fit. Right: Abundances of selected species as a function of radius predicted by the model of L1544 after $100 \mathrm{yr}$ of freeze-out.

chemical model of the core centre, could prove useful in future attempts to determine $\left[\mathrm{e}^{-}\right]$.

Another important prediction of the model is the atomic $\mathrm{D} / \mathrm{H}$ ratio, which rises from 0.1 to $\sim 0.6$ between $10^{4}$ and $10^{5}$ yr. As mentioned above, observations of molecular $\mathrm{D} / \mathrm{H}$ ratios in protostellar sources for species which are assumed to have formed on the grains, requires that the atomic $\mathrm{D} / \mathrm{H}$ ratio in the gas-phase at the time the species formed was $\geqslant 0.1$. This is discussed further in $\S 4$.

\section{1. $D_{2} C O$ in L1544: An Updated Comparison}

Another piece of observational evidence which links freeze-out with high deuteration is the study by Bacmann et al. $(2002,2003)$ of $\mathrm{CO}$ depletion and $\mathrm{D}_{2} \mathrm{CO}$ fractionation in six prestellar cores. These authors found $\mathrm{D}_{2} \mathrm{CO} / \mathrm{H}_{2} \mathrm{CO}$ ratios ranging between 0.01 and 0.1 , with the largest $\mathrm{D}_{2} \mathrm{CO}$ ratios from the sources which have the highest $\mathrm{CO}$ depletion. For $\mathrm{L} 1544$ their measured $\mathrm{D}_{2} \mathrm{CO} / \mathrm{H}_{2} \mathrm{CO}$ ratio is 0.04 and the $\mathrm{CO}$ depletion factor (compared to the canonical value of $10^{-4}$ with respect to $\mathrm{H}_{2}$ ) is 14 .

The above model requires $\mathrm{CO}$ to be depleted by $\sim 40$ to reproduce the observed $\mathrm{D}_{2} \mathrm{CO} / \mathrm{H}_{2} \mathrm{CO}$ ratio. One has to remember, though, that the above model only represents physical conditions at the centre of a prestellar core. Bacmann et al. (2002) calculate an overall density and depletion factor for each prestellar core, but, in reality, these quantities increase towards the core centres. The high $\mathrm{D}_{2} \mathrm{CO} / \mathrm{H}_{2} \mathrm{CO}$ ratios observed by Bacmann et al. may, therefore, originate in the inner regions of the prestellar cores, where the CO is heavily depleted, while the bulk of the CO emission is coming from a lower density, less depleted, envelope. In Roberts et al. (2004) the density structure of L1544 was approximated using a set of models at different densities, based on the density profile determined by Tafalla et al. (2002). Molecular abundances from each model were multiplied by the assumed path length and then summed to get a prediction for the column density through the centre of each core. This resulted in significantly better agreement: a predicted $\mathrm{D}_{2} \mathrm{CO} / \mathrm{H}_{2} \mathrm{CO}$ ratio of 0.03 for an overall $\mathrm{CO}$ depletion factor of 20 .

The chemical reaction network in the model has now been updated. Figure 2 shows the fit to the observed temperature and density profiles and the distribution of species as a function of radius at an early time point in the model. The CO abundance is initially assumed to be close to its canonical value and constant across the core. Figure 3 shows the predicted distribution of species after $10^{4} \mathrm{yr}$ and after $10^{5} \mathrm{yr}$. After $10^{4} \mathrm{yr}$ the abundance of $\mathrm{CO}$ is starting to decline at the core centre, as the higher density means freeze-out occurs more rapidly. The abundance of $\mathrm{D}_{3}^{+}$is higher closer to the core centre, and is 

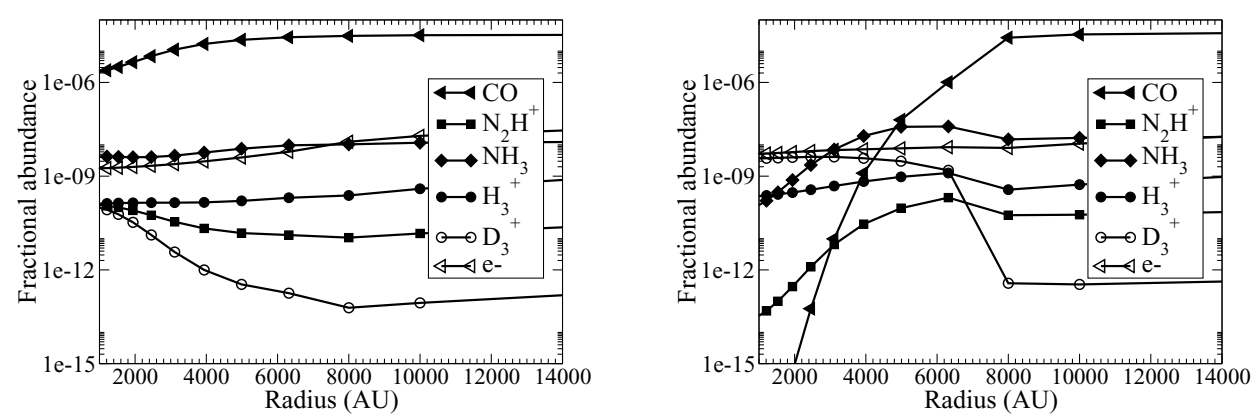

Figure 3. Abundances of selected species as a function of radius predicted by the model of L1544. Left: after $10^{4} \mathrm{yr}$; Right: after $10^{5} \mathrm{yr}$.

equal to that of $\mathrm{H}_{3}^{+}$within $\sim 1000 \mathrm{AU}$. Once $10^{5} \mathrm{yr}$ have passed the $\mathrm{CO}$ is completely frozen out at the core centre and $\left[\mathrm{D}_{3}^{+}\right] \sim\left[\mathrm{e}^{-}\right]$.

One of the major changes to the deuterium chemistry in this latest model is the way formaldehyde is fractionated. Roberts et al. (2004) pointed out that the level of fractionation might be overestimated by only considering the $\mathrm{H}_{3} \mathrm{CO}^{+}$form of protonated formaldehyde (along with its deuterated analogues), rather than the lower energy $\mathrm{H}_{2} \mathrm{COH}^{+}$isomer. If $\mathrm{D}_{3}^{+}$reacts with $\mathrm{H}_{2} \mathrm{CO}$ to form $\mathrm{H}_{2} \mathrm{DCO}^{+}$, then $\mathrm{H}_{2} \mathrm{DCO}^{+}$can recombine with electrons to produce $\mathrm{HDCO}$. The $\mathrm{HDCO}$ also reacts with $\mathrm{D}_{3}^{+}$, producing $\mathrm{HD}_{2} \mathrm{CO}^{+}$and then $\mathrm{D}_{2} \mathrm{CO}$. Using this scheme, the fractionation in $\mathrm{D}_{2} \mathrm{CO}$ will track that of $\mathrm{D}_{3}^{+}$and will, therefore, be enhanced in regions where $\mathrm{CO}$ is depleted.

Recent quantum chemical calculations by Osamura et al. (2005), however, suggest that the above route to $\mathrm{D}_{2} \mathrm{CO}$ is very inefficient. Instead, when $\mathrm{D}_{3}^{+}$reacts with $\mathrm{H}_{2} \mathrm{CO}$, $\mathrm{H}_{2} \mathrm{COD}^{+}$is formed, which then dissociates back to $\mathrm{H}_{2} \mathrm{CO}$, and similarly for HDCO. Instead of $\mathrm{H}_{2} \mathrm{CO}$ being converted to $\mathrm{D}_{2} \mathrm{CO}, \mathrm{D}_{2} \mathrm{CO}$ forms via the analogue of the reaction which forms the bulk of the $\mathrm{H}_{2} \mathrm{CO}$ :

$$
\mathrm{CHD}_{2}+\mathrm{O} \longrightarrow \mathrm{D}_{2} \mathrm{CO}+\mathrm{H} .
$$

This does result in a some enhancement of the $\mathrm{D}_{2} \mathrm{CO} / \mathrm{H}_{2} \mathrm{CO}$ ratio, since the $\mathrm{CHD}_{2} / \mathrm{CH}_{3}$ ratio is enhanced through the $\mathrm{CHD}_{2}^{+}$ion (reactions (2.4) and (2.5)). The energy barrier for destruction of deuterated $\mathrm{CH}_{3}^{+}$by $\mathrm{H}_{2}$ is higher than for $\mathrm{H}_{3}^{+}$, so this process is efficient up to temperatures of $\sim 50 \mathrm{~K}$, but, as shown by Millar et al. (2000), the depletion of neutral species does not have the same dramatic effect on $\mathrm{CH}_{3}^{+}$fractionation that it does for $\mathrm{H}_{3}^{+}$.

Instead of simply presenting column densities through the core centre, these latest model results are used to calculate beam-averaged column densities, which should allow a better comparison with observations. Averaging over a $10^{\prime \prime}$ beam, at the distance of L1544 (220 pc), for times between $10^{4}$ and $10^{5} \mathrm{yr}$, the column density of CO decreases from $10^{18}$ to $4 \times 10^{17} \mathrm{~cm}^{-2}$, while that of $\mathrm{H}_{2} \mathrm{D}^{+}$is $2-5 \times 10^{13} \mathrm{~cm}^{-2}$, consistent with the observations of $N(\mathrm{CO}) \sim 9 \times 10^{17} \mathrm{~cm}^{-2}$ (Bacmann et al. 2002) and $N\left(\mathrm{H}_{2} \mathrm{D}^{+}\right)=$ $5 \times 10^{13} \mathrm{~cm}^{-2}$ (Caselli et al. 2003). Figure 4 shows predictions for the deuterium fractionation in selected molecules, based on column density ratios. For $\mathrm{H}_{2} \mathrm{D}^{+}$and $\mathrm{HD}_{2}^{+}$, after a few times $10^{4} \mathrm{yr}$, the ratios are in agreement with the one-point model and with the observations (i.e. $\left[\mathrm{HD}_{2}^{+}\right] \sim\left[\mathrm{H}_{2} \mathrm{D}^{+}\right] \sim\left[\mathrm{H}_{3}^{+}\right]$), but the highest predicted $\mathrm{D}_{2} \mathrm{CO} / \mathrm{H}_{2} \mathrm{CO}$ ratio is now 0.001. It is worth noting, however, that $N\left(\mathrm{D}_{2} \mathrm{CO}\right)$ is a few times $10^{12} \mathrm{~cm}^{-2}$, compared with the observed value of $9 \times 10^{11} \mathrm{~cm}^{-2}$ (Bacmann et al. 2003), so the 


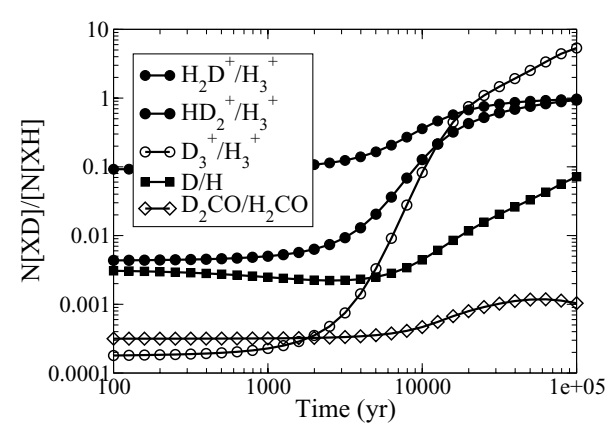

Figure 4. Molecular D/H ratios evolving over time, predicted for a core with the structure of L1544, that one would expect to observe with a $10^{\prime \prime}$ beam.

problem is not that the models don't produce enough $\mathrm{D}_{2} \mathrm{CO}$, rather that they produce too much $\mathrm{H}_{2} \mathrm{CO}$.

One possible solution could be the inclusion of surface chemistry in the models. The grains do not merely act as passive repositories for the species freezing out, they also catalyse chemical reactions. This has long been known as the only efficient way to produce $\mathrm{H}_{2}$ in dark clouds, while the enhancement of $\mathrm{H}_{2} \mathrm{O}, \mathrm{H}_{2} \mathrm{CO}, \mathrm{CH}_{3} \mathrm{OH}, \mathrm{NH}_{3}$ and $\mathrm{H}_{2} \mathrm{~S}$ observed in protostellar sources indicates that atoms and unsaturated molecules are readily hydrogenated. It is expected, therefore, that the relative abundances of $\mathrm{H}_{2} \mathrm{CO}, \mathrm{HDCO}$, and $\mathrm{D}_{2} \mathrm{CO}$ formed from the solid-state $\mathrm{CO}$, will depend upon the relative amounts of $\mathrm{H}$ and $\mathrm{D}$ landing on the grains. With the high atomic $\mathrm{D} / \mathrm{H}$ ratio predicted by the above models, it is possible to produce a high $\mathrm{D}_{2} \mathrm{CO} / \mathrm{H}_{2} \mathrm{CO}$ ratio on the surface, and, although desorption processes at low temperatures are not well understood, there could be mechanisms for returning some of this formaldehyde to the gas-phase. There are two problems with this hypothesis, though. The first is that there is a significant abundance of $\mathrm{H}_{2} \mathrm{CO}$ formed in the gas-phase, and unless larger amounts were formed on and desorbed from the surface, this would dilute the surface fractionation. The second is that if significant amounts of neutral species continuously desorb from the surface, they will react with $\mathrm{H}_{3}^{+}$ and its analogues, competing with the deuterium fractionation reactions. This will reduce the molecular $\mathrm{D} / \mathrm{H}$ ratios including, crucially, the atomic $\mathrm{D} / \mathrm{H}$ ratio, meaning that the fractionation produced on the grain-surfaces may not then reach the level required.

\section{Low-Mass Protostars}

The regions surrounding low-mass protostars are more complex, containing multiple temperature and density components, and the chemistry may have been impacted by shocks from the protostellar outflow as well as thermal heating. Despite the higher temperatures, which would seem to preclude chemical fractionation via reactions $(2.1)-(2.3)$, molecular $\mathrm{D} / \mathrm{H}$ ratios can be very high. In fact, the highest ratios measured for formaldehyde, methanol and hydrogen sulphide are observed towards these regions. The thing which is immediately obvious about this list of species is they are typically those which do not form efficiently via ion-molecule chemistry or are seen to have abundance jumps close to the protostar (e.g. Maret et al. 2004; Parise et al. 2005), indicating that they have evaporated from grain surfaces. The $\mathrm{DCO}^{+} / \mathrm{HCO}^{+}$ratios in these sources are typically lower than in the prestellar cores (see Table 1), supporting the assumption that, at higher temperatures, fractionation is not dominated by deuterated $\mathrm{H}_{3}^{+}$.

It is now generally accepted that the deuterium fractionation in the protostellar cores was set during the cold, dense prestellar phase, when the atomic $\mathrm{D} / \mathrm{H}$ ratio in the gas was 
very high. Methanol and hydrogen sulphide are probably the best molecules to use to probe deuterium fractionation processes on grain surfaces. Formaldehyde and ammonia, whose deuterated analogues have also been widely observed in protostellar sources, can form via both gas-phase and grain-surface chemistry so the resulting fractionation in the warm gas will depend on the proportions coming from each route.

Current protostellar models usually assume the initial grain-mantle composition, rather than calculating it via a chemical model. At $\mathrm{t}=0 \mathrm{yr}$ everything is frozen onto the grains, and the star is assumed to 'switch-on', causing either instantaneous or gradual heating which desorbs species from grains. If the model predicts that the molecular $\mathrm{D} / \mathrm{H}$ ratios remain constant for a long time after evaporation, then observations can be used to directly determine the surface fractionation, and estimate the temperature at which the mantles formed. This has been done for $\mathrm{HDS} / \mathrm{H}_{2} \mathrm{~S}$ near high-mass protostars (Hatchell et al. 1999). If, instead, the fractionation changes after evaporation (as appears to be the case for certain isotopomers of methanol) then the observed molecular $\mathrm{D} / \mathrm{H}$ ratios can be used to estimate the time since mantle evaporation (Osamura et al. 2004).

\section{Conclusions and Future Directions}

Since the last Astrochemistry Symposium (IAUS 197, Korea, 1999) observations of enormous levels of deuterium fractionation have been made, proving a challenge for the models to explain (e.g. Loinard et al. 2001; Ceccarelli 2002; Parise et al. 2002). One of the major successes of the past three years, therefore, is the revision of the low-temperature chemical fractionation mechanism to include multiply deuterated forms of $\mathrm{H}_{2} \mathrm{D}^{+}$(Lis et al. 2002; Phillips \& Vastel 2003). This leads naturally to high molecular D/H ratios in low-temperature gas, but also goes a long way towards explaining the ratios in protostellar sources with the prediction of a very high atomic $\mathrm{D} / \mathrm{H}$ ratio which lasts for $\sim 10^{5} \mathrm{yr}$ (Roberts et al. 2003). It also suggests a potentially useful way to measure the ionisation fraction at the centre of a prestellar core in the very last stages before the star forms (Flower et al. 2004).

Obviously, there are improvements which still need to be made to the models before we can fully exploit the potential of molecular $\mathrm{D} / \mathrm{H}$ ratios to trace conditions throughout the different stages of star-formation. Most of these uncertainties involve the gas-grain interaction and the grain-surface chemistry. To improve the models further we need to include desorption mechanisms at low-temperatures, and accurate binding energies for species on different surfaces are required. Many of the parameters needed for detailed surface chemistry models are also uncertain, although a lot of theoretical and experimental work is being carried out, and is described elsewhere in this volume.

For more accurate gas-phase fractionation models, we do need to confirm the rate coefficients for the fractionation reactions at $10 \mathrm{~K}$, and for the dissociative recombination of $\mathrm{H}_{3}^{+}$and its deuterated analogues. Experiments to determine whether the branching ratios for dissociative recombination of deuterated ions are statistical, or if the heavier deuterium is more likely to be retained in the larger products, would also be extremely useful.

\section{References}

Bacmann, A., Lefloch, B., Ceccarelli, C., Castets, A., Steinacker, J., \& Loinard, L. 2002, A\&A 389, L6

Bacmann, A., Lefloch, B., Ceccarelli, C., Steinacker, J., Castets, A., \& Loinard, L. 2003, Ap. J. 585, L55 
Bell, M.B., Avery, L.W., Matthews, H.E., Feldman, P.A., Watson, J.K.G., Madden, S.C., \& Irvine, W.M. 1988, Ap. J. 326, 924

Brown, P.B., \& Millar, T.J. 1989, MNRAS 237, 661

Caselli, P., Walmsley, C.M., Tafalla, M., Dore, L., \& Myers, P.C. 1999, Ap. J. 523, L165

Caselli, P., van der Tak, F.F.S., Ceccarelli, C., \& Bacmann, A. 2003, A\&A 403, L37

Caselli, P., Walmsley, C.M., Zucconi, A., Tafalla, M., Dore, L., \& Myers, P.C. 2002, Ap. J. 565, 344

Ceccarelli, C. 2002, P\&S SS 50, 1267

Ceccarelli, C., Loinard, L., Castets, A., Tielens, A.G.G.M., Caux, E., Lefloch, B., \& Vastel, C. 2001, A\&A 372, 998

Flower, D.R., Pineau des Forêts, G., \& Walmsley, C.M. 2004, A $\& A$ 427, 887

Gerin, M., Combes, F., Wlodarczak, G., Encrenaz, P., \& Laurent, C. 1992, A\&3A 253, L29

Gerin, M., Pearson, J.C., Roueff, E., Falgarone, E., \& Phillips, T.G. 2001, Ap. J. 551, L193

Guélin, M., Langer, W.D., \& Wilson, R.W. 1982, A\&\&A 107, 107

Hatchell, J. 2003, A\&SA 403, L25

Hatchell, J., Roberts, H., \& Millar, T.J. 1999, A\&A 346, 227

Herbst, E. 2000, Phil. Trans. R. Soc. Lond. A. 358, 2523

Hirota, T., Ikeda, M., \& Yamamoto, S. 2003, Ap. J. 594, 859

Howe, D.A., Millar, T.J., Schilke, P., \& Walmsley, C.M. 1994, MNRAS 267, 59

Linsky, J.L. 1998, Space Sci. Rev. 84, 285

Lis, D.C., Roueff, E., Gerin, M., Phillips, T.G., Coudert, L.H., van der Tak, F.F.S., \& Schilke, P. 2002, Ap. J. 571, L55

Loinard, L., Castets, A., Ceccarelli, C., Caux, E., \& Tielens, A.G.G.M. 2001, Ap. J. 552, L163

Loinard, L., Castets, A., Ceccarelli, C., et al. 2002, P\&SSS, 50, 1205

MacLeod, J.M., Avery, L.W., \& Broten, N.W. 1981, Ap. J. 251, L33

Maret, S., Ceccarelli, C., Caux, E., et al. 2004, A\&A 416, 577

Marcelino, N., Cernicharo, J., Roueff, E., Gerin, M., \& Mauersberger, R. 2005, Ap. J. 620, 308

Millar, T.J., Bennett, A., \& Herbst, E. 1989, Ap. J. 340, 906

Millar, T.J., Roberts, H., Markwick, A.J., \& Charnley, S.B. 2000, Phil. Trans. R. Soc. Lond. A. 358,2359

Minowa, H., Satake, M., Hirota, T., Yamamamoto, S., Ohishi, M., \& Kaifu, N. 1997, Ap. J. 491, L63

Osamura, Y., Roberts, H., \& Herbst, E. 2004, A\&\&A 421, 1101

Osamura, Y., Roberts, H., \& Herbst, E. 2005, Ap. J. 621, 348,

Pagani, L., Pardo, J.-R., Apponi, A.J., Bacmann, A., \& Cabrit, S. 2005, A\&\&A 429, 181

Parise, B., Ceccarelli, C., Tielens, A.G.G.M., et al. 2002, A\&SA 393, L49

Parise, B., Castets, A., Herbst, E., et al. 2004, A\& A 416, 159

Parise, B., Caux, E., \& Castets, A., et al. 2005, A $\& A 431,547$

Phillips, T.G., \& Vastel, C. 2003, in Chemistry as a Diagnostic of Star Formation, ed. C.L. Curry \& M. Fich, NRC Press, Ottowa, Canada, p. 3

Roberts, H., \& Millar, T.J. 2000, A\&A 361, 388

Roberts, H., Herbst, E., , \& Millar, T.J. 2003, Ap. J. 591, L41

Roberts, H., Herbst, E., \& Millar, T.J. 2004, A\& $A$ 424, 905

Roueff, E., Tiné, S., Coudert, L.H., Pineau des Forêts, G., Falgarone, E., \& Gerin, M. 2000, A\&GA 354, L63

Saito, S., Ozeki, H., Ohishi, M., \& Yamamoto, S. 2000, Ap. J. x535, 227

Shah, R.Y., \& Wootten, A. 2001, Ap. J. 554, 933

Sonneborn, G., Tripp, T. M., Ferlet, R., Jenkins, E.B., Sofia, U.J., Vidal-Madjar, A., \& Wozniak, P. $2000, A p . J .545,277$

Stark, R., van der Tak, F.F.S., \& van Dishoeck, E.F. 1999, Ap. J. 521, L67

Stantcheva, T., \& Herbst, E. 2003, MNRAS 340, 983

Tafalla, M., Myers, P.C., Caselli, P., Walmsley, C.M., \& Comito, C. 2002, Ap. J. 569, 815

Tiné, S., Roueff, E., Falgarone, E., Gerin, M., \& Pineau des Forêts, G. 2000, A\& $A$ 356, 1039

Turner, B.E. 1989, Ap. J. 347, L39

Turner, B.E. 2001, ApJS 136, 579 
van der Tak, F.F.S., Schilke, P., Müller, H.S.P., Lis, D.C., Phillips, T.G., Gerin, M., \& Roueff, E. 2002, A\&A 388, L53

van Dishoeck, E.F., Blake, G.A., Jansen, D.J., \& Groesbeck, T.D. 1995, Ap. J. 447, 760

Vastel, C., Phillips, T.G., Ceccarelli, C., \& Pearson, J. 2003, Ap. J. 593, L97

Vastel, C., Phillips, T.G., \& Yoshida, H. 2004, Ap. J. 606, L127

Wootten, A. 1987, in Astrochemistry, ed. M.S. Vardya S.P. Tarafdar (Reidel, Dordrecht) p. 311

\section{Discussion}

RAWLings: The potential surfaces (and the dissociative recombination rates) for the deuterated forms of $\mathrm{H}_{3}^{+}$are highly uncertain. How does this affect the modeled $\mathrm{D}_{3}^{+}$, $\mathrm{HD}_{2}^{+}, \mathrm{H}_{2} \mathrm{D}^{+}$abundances?

RoBERTs: For $\mathrm{H}_{2} \mathrm{D}^{+} / \mathrm{H}_{3}^{+}$and $\mathrm{HD}_{2}^{+} / \mathrm{H}_{2} \mathrm{D}^{+}$the destruction is dominated by reaction of the deuterated ion with $\mathrm{HD}$ (as the electron fraction is so low at densities of $10^{6} \mathrm{~cm}^{-3}$ ), so the dissociative recombination rate does not have a major effect. For $\mathrm{D}_{3}^{+} / \mathrm{HD}_{2}^{+}$, however, recombination with electrons is its only destruction channel (in the last stage of depletion) so here, yes, that rate is an important parameter.

HERBST: (1) For protostellar cores, should we not distinguish between inner (warmer) and outer (cooler) regions? (2) How will models be affected by the new measurement that $\mathrm{CH}_{3} \mathrm{OH}_{2}^{+}+e^{-}$does not produce $\mathrm{CH}_{3} \mathrm{OH}+\mathrm{H}$ ?

RoBerts: (1) Yes. I am currently working on this. (2) If methanol is destroyed by dissociated recombination, then the selective fall in methanol isotopologues wih an -OD group may no longer occur. In that case we would have to look for alternate (possibly grain-surface) processes (see talk by Watanabe).

WALMSLEY: Do you assume "normal ISM grains" in your models? This can have a considerable effect on the degree of $\mathrm{D}$ fractionation.

RoBerts: Yes, we only consider "normal grains". The nature of the grain surfaces is something we should consider in the future. 